\title{
Combination of Tung oil and Natural Rubber Latex in PVA as Water Based Coatings for Paperboard Application
}

\author{
Apichaya Jianprasert ${ }^{1, a}$, Pathavuth Monvisade ${ }^{2}$ and Masayuki Yamaguchi ${ }^{3}$ \\ ${ }^{1}$ College of Nanotechnology, King Mongkut's Institute of Technology Ladkrabang, Thailand \\ ${ }^{2}$ Polymer Synthesis and Functional Materials Research Unit, Department of Chemistry, Faculty of Science, \\ King Mongkut's Institute of Technology Ladkrabang, Thailand \\ ${ }^{3}$ School of Materials Science, Japan Advanced Institute of Science and Technology, Japan
}

\begin{abstract}
This research is focused on the preparation of the PVA/TO/NRL coatings for paperboard by using poly(vinyl alcohol) (PVA) as substance and blending with Tung oil (TO) and/or natural rubber latex (NRL) in order to enhance water resistance and dynamic mechanical properties. The effects of TO: NRL ratios on the structures were investigated by water resistance property and dynamic mechanical thermal analysis (DMA). The results showed that the water resistance property was improved by crosslinking of TO and film forming of NRL. The PVA/TO/NRL coating containing both TO and NRL gave better thermal behavior than those with only TO or NRL. For paperboard application, the PVA/TO/NRL coatings were applied on the paperboard to study water affinity and absorption rate on the coated surface. The rate of contact angle change of water on coated paperboards decreased depending on the ratios of TO and NRL.
\end{abstract}

\section{Introduction}

Coating \& Laminate technologies are the regular stage for protection of the surface of wood and paperboard products[1]. Among various coating polymers, PVA is an important water-soluble synthetic polymer, which has been widely used as coating agents $[2,3]$. However, it has poor resistance to water. To improve the water resistance property, PVA blends with other hydrophobic polymer have been focused. Natural rubber latex (NRL), a natural high elasticity material, is widely used in the manufacture of thin film products [4]. Although the NRL provides better water resistance, it must be vulcanized by sulphur or peroxide system with high temperature $\left(>100^{\circ} \mathrm{C}\right)$. Tung oil (TO), a drying oil, is alternatively used as environment-friendly coating for decades because it can crosslink to a solid film rapidly after exposure to air at even room temperature[5,6]. Although TO can crosslink at lower curing temperature, it is relatively expensive and its crosslinked form is more brittle than that of NRL. Combination of NRL and TO is an easy way to improve water resistance of PVA including the reduction in curing temperature. Therefore this work aims to create a water-based coatings based on PVA. TO and/or NRL at various NRL:TO ratios were applied in the coatings. Moreover, redox catalytic system, a couple of potassium persulfate (KPS) and sodium thiosulfate[7,8], was also used to lowering curing temperature for crosslinking reaction. The effects of NRL:TO ratios in PVA/TO/NRL coatings were studied by FTIR and DMA. The water resistance property of

\footnotetext{
${ }^{\mathrm{a}}$ Corresponding author: kjapitch@gmail.com
}

PVA/TO/NRL coatings was also investigated. Finally, the PVA/TO/NRL coatings were applied on paperboard in order to study water affinity and absorption rate by contact angle measurement of water tests.

\section{Experimental}

\subsection{Preparation and characterization of PVA/TO/NRL coatings}

The PVA solution (15 wt\%) in PVA/TO/NRL coatings was fixed at $85 \%$ by weight. Natural rubber latex (NRL) and/or Tung oil (TO) was added to the PVA solution with the weight ratios of (NRL:TO) 15:0 (R15T0), 10:5 (R10T5), 5:10 (R5T10) and 0:15 (R0T15) under continuous mixing to obtain a homogeneous mixture. Redox catalyst (potassium persulfate and sodium thiosulfate) was then filled into the mixture and stirred vigorously for $15 \mathrm{~min}$. The PVA/TO/NRL coatings were later cast into the Petri dish and dried at $50{ }^{\circ} \mathrm{C}$ in an oven. The thickness of the PVA/TO/NRL coating sheets measured at five different points using a micrometer was approximately $300 \pm 50 \mu \mathrm{m}$. After that PVA/TO/NRL coatings were characterized by FTIR and DMA.

\subsection{Water resistance of PVA/TO/NRL coatings}

The PVA/TO/NRL coating sheets were cut into a rectangular shape $(1.5 \times 2 \mathrm{~cm})$. Each of the specimens was 


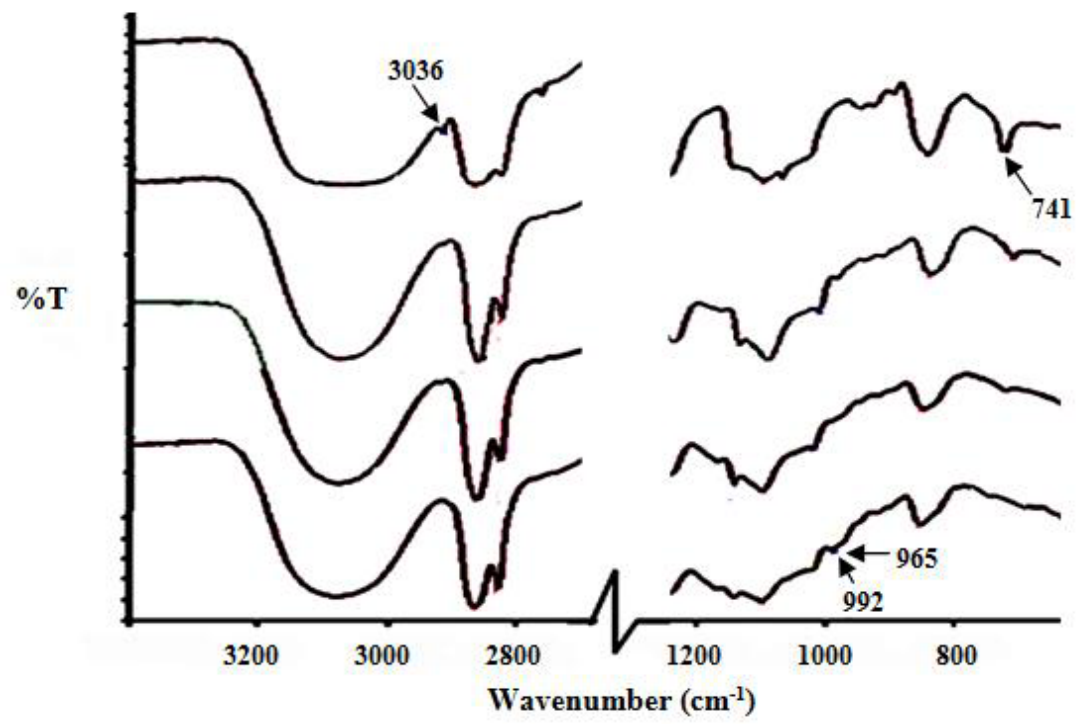

Figure 1. FTIR spectra of PVA/TO/NRL coatings using various NRL:TO ratios

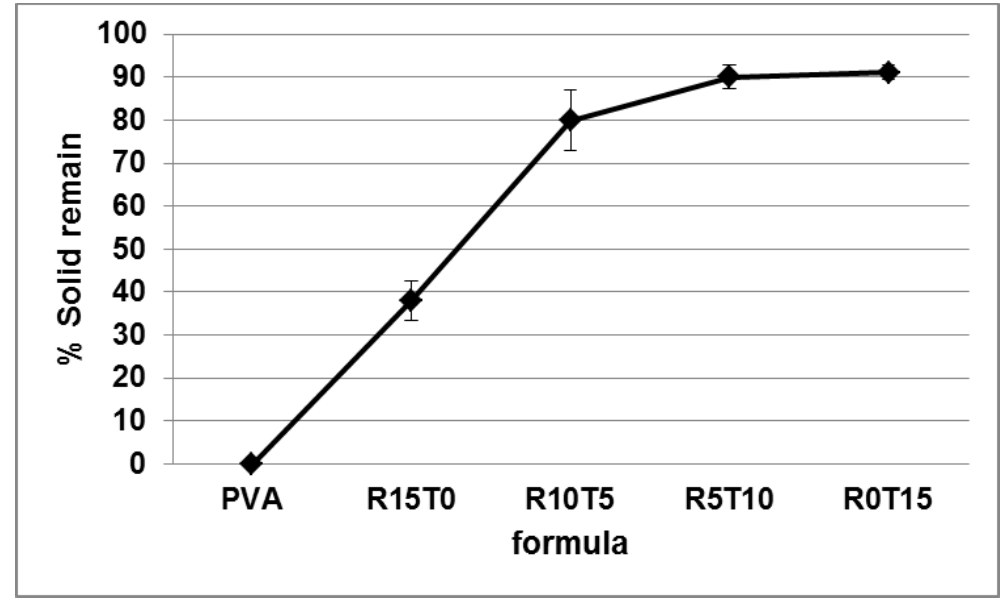

Figure 2. Solid remain (\%) of PVA/TO/NRL coatings using various NRL:TO ratios after immersion in water at $25^{\circ} \mathrm{C}$ for $24 \mathrm{~h}$.

weighed and immersed in water for $24 \mathrm{~h}$. Finally, the specimen was dried to constant weight in an oven. The percentage of solid remains was calculated as follows:

$$
\% \text { Solid remain }=\left(W_{2} / W_{1}\right) \times 100
$$

Where $\mathrm{W}_{1}$ is the dry weight $(\mathrm{g})$ before immersion in water and $\mathrm{W}_{2}$ is the dry weight $(\mathrm{g})$ after immersion in water for $24 \mathrm{~h}$.

\subsection{Initial contact angle and dynamic change of contact angle}

The contact angle of water on the surface and dynamic contact angle change of coated samples were measured with a contact angle analyzer (model OCA 20, Dataphysics instruments) at room temperature. A distilled water drop of $c a .30 \mu \mathrm{L}$ was placed on surfaces of the uncoated or coated samples using micro-syringe. Dynamic contact angle change was measured by recording the contact angle change of a water drop with time within $300 \mathrm{~s}$ at room temperature.

\section{Results and Discussion}

\subsection{FTIR characterization}

The FTIR spectra of PVA/TO/NRL coatings as a function of NRL:TO ratios are shown in Fig. 1. The $-\mathbf{C}=\mathbf{C}-\mathbf{H}$ vibration in the structures of TO and NRL was allowed to follow the formation of crosslinking reaction. For R0T15, the pattern of the characteristic peaks related to $-\mathrm{C}=\mathbf{C}$ $\mathbf{H}$ vibrations of TO $\left(3012 \mathrm{~cm}^{-1}\right.$ for $-\mathbf{C}=\mathbf{C}-\mathbf{H}$ stretching, $992 \mathrm{~cm}^{-1}$ for conjugated trans: trans $-\mathrm{C}=\mathbf{C}-\mathbf{H}$ bending and $965 \mathrm{~cm}^{-1}$ for conjugated cis: trans $-\mathbf{C}=\mathbf{C}-\mathbf{H}$ bending) [9] disappeared. It implies that the crosslinking network in the films would occur via the addition reaction at the double bonds of TO. On the contrary, R15T0 still showed characteristic peaks corresponding to 

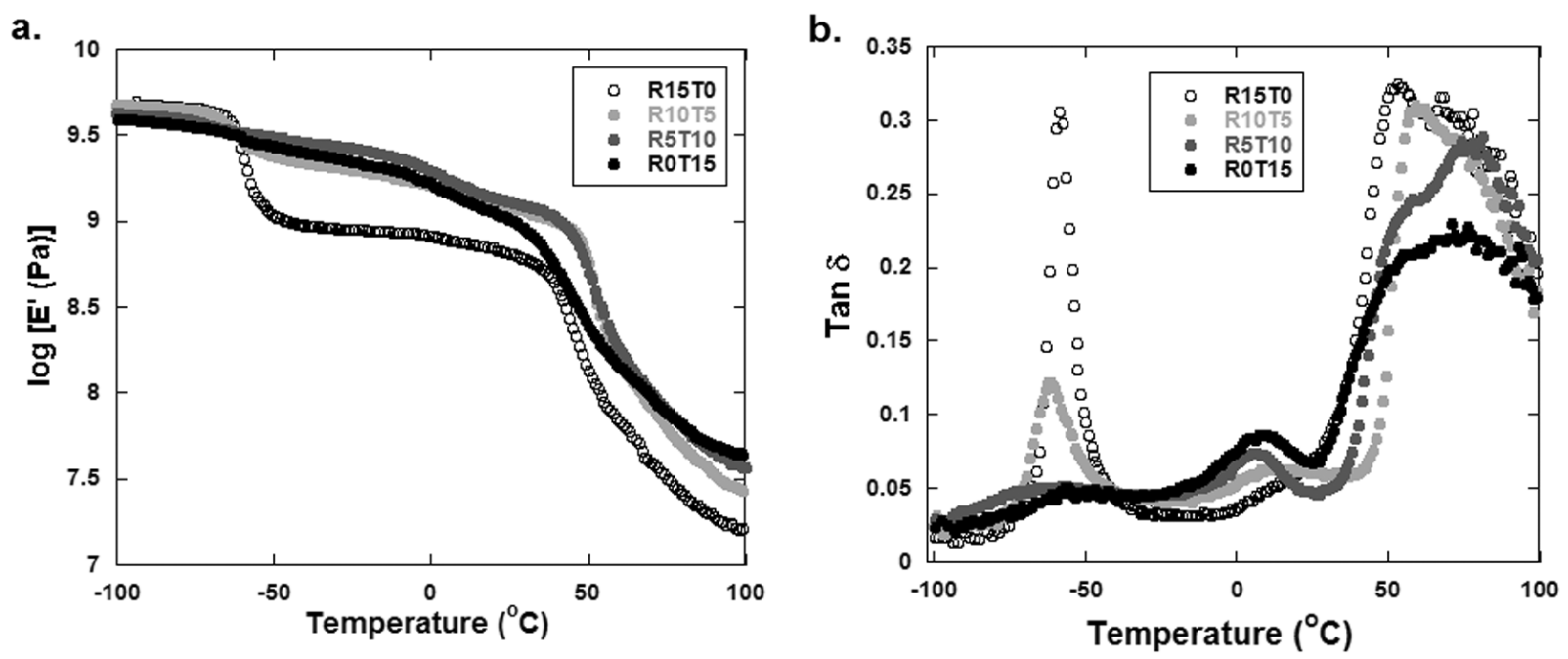

Figure 3. Temperature dependence of (a) storage modulus (E'), and (b) tan $\delta$ of PVA/TO/NRL coatings

double bonds of NRL at $3036 \mathrm{~cm}^{-1}(-\mathrm{C}=\mathbf{C}-\mathbf{H}$ stretching $)$ and $741 \mathrm{~cm}^{-1}(-\mathrm{C}=\mathbf{C}-\mathbf{H}$ bending $)$. It is suggested that the crosslinking reaction at double bonds of NRL did not predominate. In case of R10T5 and R5T10, only the characteristic peaks of TO depleted whereas those of NRL remained, suggesting that the crosslinking reaction of TO was predominant.

\subsection{Water resistance of PVA/TO/NRL coatings}

The water resistance of the coating adhesive was investigated in terms of the \% solid remain as shown in Fig.2. Considering to NRL and TO structures, they have hydrophobic structures. Thus the addition of NRL and/or TO could improve the water resistance of PVA. As observed in Fig.2, the \% solid remain values of R15T0 were higher than that of neat PVA. In addition, the presence of TO dramatically increased the \% solid remain values compared with those of neat PVA and R15T0. This is because TO could crosslink to be the network structures at the conjugated double bonds of TO through free radical mechanism[10]. Therefore, PVA and NRL chains were retained within the crosslinked TO structures. This caused PVA and NRL in R10T5, R5T10 and R0T15 to have more difficulty to dissolve in water than R15T0.

\subsection{Mechanical thermal property of PVA/TO/NRL coatings}

The $\tan \delta$ and storage modulus (E') of PVA/TO/NRL coatings in various NRL:TO ratios were investigated by DMA, as shown in Fig. 4(a) and (b), respectively. The $\mathrm{T}_{\mathrm{g}}$ of PVA in PVA/TO/NRL coatings increased with increasing TO content. This is because when TO crosslinked, NRL and PVA chains were retained within the crosslinked TO structures resulting in the restriction movement of NRL and PVA chains. In case of E', the storage modulus of R15T0 showed the lowest value than that of other PVA/TO/NRL coatings because NRL could not crosslink at relatively low curing temperature. The combination of TO and NRL in the coatings displayed a high level of E' compared to R15T0 and R0T15. This phenomenon was due to the crosslinked structures of TO within the PVA/TO/NRL coatings resulting to enhancing storage modulus. In addition, the intensities of $\tan \delta$ peak of PVA in PVA/TO/NRL coatings with higher TO content exhibited a depression trend compared to that of the R15T0 coating because of the increase in network chain by the crosslinking reaction.

\subsection{Initial contact angle and dynamic change of contact angle}

The water contact angle of the uncoated paperboard, and coated paperboard with PVA/TO/NRL coatings was investigated. An uncoated paperboard showed water contact angle of $105.2 \pm 8.2^{\circ}$. In case of the coated paperboard, the initial contact angles of water were in the range of $59.6-104.7^{\circ}$ lower than that of the uncoated paperboard. The lower level in water contact angle by PVA/TO/NRL coatings indicates the higher wetting ability due to the change in the chemical compositions on the surface. Moreover, the synergy of TO and NRL in coating adhesive presented the relatively high in water contact angle, especially when R5T10 was applied. It is reasonable because TO and NRL are hydrophobic structures and the crosslinked TO resisted to water absorption.

The rate of contact angle change $(\mathrm{k})$ of water on paperboards decreased from $0.095 \mathrm{~s}^{-1}$ of the uncoated paperboard to the range of $0.045-0.069 \mathrm{~s}^{-1}$ of the coated paperboards. A slightly decrease in the $\mathrm{k}$ values pointed at better water barrier of the surface because of chemical compositions of $\mathrm{PVA} / \mathrm{TO} / \mathrm{NRL}$ coatings. The results demonstrated that all PVA/TO/NRL coatings can modify the surfaces of paperboards to yield better water affinity with lower absorption rate. 


\section{Conclusion}

The PVA/TO/NRL coatings were prepared for paperboard coatings by using low curing temperature. The crosslinking reaction of TO and NRL in PVA/TO/NRL coatings were studied by FTIR and DMA, respectively. The results confirmed that TO can crosslink resulting in the improvement of water resistance and thermal property of PVA. In addition, the synergy of TO and NRL in PVA/TO/NRL coatings gave better thermal behavior than those using only TO or NRL. Moreover, the coated paperboards by the PVA/TO/NRL coatings showed the water affinity and the decrease in water absorption rates of paperboard surface.

\section{Acknowledgements}

This work was financially supported by the Royal Golden Jubilee Ph.D. Program, Thailand Research Fund and the College of Nanotechnology, King Mongkut's Institute of Technology Ladkrabang, Bangkok, Thailand. The authors would like to thank the Scientific Instruments Service Center, Faculty of Science, King Mongkut's Institute of Technology Ladkrabang, Thailand, for contact angle analyser and FTIR. Thanks also to the School of Material Science, Japan Advanced Institute of Science and Technology (JAIST) for DMA.

\section{References}

1. A.A. Tracton, Coatings Technology Handbook, 779780 (2006)

2. T. Schuman, M. Wikström, M. Rigdahl, Surf. Coat. Tech. 183, 96-105 (2004)

3. T. Lamminmäki, J. Kettle, P. Puukko, J. Ketoja, P. Gane, Nord. Pulp. Pap. Res. J 25, 380-390 (2010)

4. W. Simchareona, T. Amnuaikita, P. Boonmea, Procedia. Chem. 4, 308-312 (2012)

5. X. Yang, S. Zhang, W. Li, Prog. Org. Coat. 85, 216$220(2015)$

6. J. T. P. Derksen, F. P. Cuperus, P. Kolster, Prog. Org. Coat. 27, 45-53 (1996)

7. W. Bai, L. Zhang, R. Bai, Macromol. Rapid. Comm. 29, 562-566 (2008)

8. S. Riyajan, S. Chaiponban, K. Tanbumrung, Chem. Eng. J. 153, 199-205 (2009)

9. Y. Wang, Q. Wang, W.E. Artz, G.W. Padua, J. Agr. Food. Chem. 56, 3043-3148 (2008)

10. Z.O. Oyman, W. Ming, R. van der Linde, Prog. Org. Coat. 54, 198-204 (2005) 